\title{
ADUBAÇÃO QUÍMICA E ORGÂNICA NA RECUPERAÇÃO DA FERTILIDADE DE SUBSOLO DEGRADADO E NA MICORRIZAÇÃO DO Stryphnodendron polyphyllum ${ }^{(1)}$
}

\author{
Hemerson Fernandes Calgaro ${ }^{(2)}$, Walter Veriano Valério Filho ${ }^{(3)}$, \\ Sueli da Silva Aquino( ${ }^{(2)}$, Kátia Luciene Maltoni ${ }^{(4)}$ \& Ana Maria \\ Rodrigues Cassiolato ${ }^{(4)}$
}

\begin{abstract}
RESUMO
Com a construção da Usina Hidrelétrica de Ilha Solteira no final da década de 1960, algumas áreas foram desmatadas, servindo como "área de empréstimo". Os solos dessas áreas foram retirados e estas adquiriram características físicas, químicas e biológicas distantes das ideais. Este trabalho objetivou avaliar os efeitos da aplicação da adubação química e orgânica na fertilidade do subsolo degradado e micorrização de Stryphnodendron polyphyllum (barbatimão). A área localiza-se na Fazenda de Ensino, Pesquisa e Extensão (FEPE) da UNESP/Ilha Solteira, em Selvíria - MS. Com delineamento de blocos ao acaso, em parcelas de $250 \mathrm{~m}^{2}$ (10 x $25 \mathrm{~m})$, foram avaliados 10 tratamentos: testemunha; calagem; adubação $\mathrm{N}+\mathrm{P}$; calagem $+\mathbf{N}+\mathbf{P} ; \mathbf{N}+\mathbf{P}+$ aguapé; $\mathbf{N}+\mathbf{P}+$ bagaço de cana; $\mathbf{N}+\mathbf{P}+$ aguapé + bagaço de cana; calagem $+\mathrm{N}+\mathrm{P}+$ aguapé; calagem $+\mathrm{N}+\mathrm{P}+$ bagaço de cana; e calagem + $\mathrm{N}+\mathbf{P}+$ bagaço de cana + aguapé. Avaliaram-se as características químicas do solo e o crescimento do barbatimão em cinco períodos (junho, agosto, novembro e dezembro de 2005 e março de 2006), e a micorrização, em março de 2006. Observouse que o subsolo continuou apresentando caráter ácido e pobreza em nutrientes após um ano de avaliação. O crescimento da planta aumentou ao longo do período, com os maiores valores para os tratamentos que receberam calagem, adubação e resíduos orgânicos, especialmente o aguapé. A colonização micorrízica foi influenciada positivamente pela presença de resíduos orgânicos, e o número de esporos, pelos tratamentos com presença de aguapé.
\end{abstract}

Termos de indexação: área de empréstimo, cerrado, aguapé, bagaço de cana-deaçúcar.

\footnotetext{
(1) Parte da Tese de Mestrado do primeiro autor apresentada ao Programa de Pós-Grauação em Agronomia da Universidade Estadual Paulista - UNESP. Recebido para publicação em junho de 2007 e aprovado em novembro de 2007.

(2) Doutorando em Produção Vegetal, Universidade Estadual Paulista - UNESP. Caixa Postal 31, CEP 15385-000 Ilha Solteira (SP). Bolsista CNPq.

(3) Professor do Departamento de Matemática, UNESP. E-mail: wvvf@fqm.feis.unesp.br

(4) Professora do Departamento de Fitossanidade, Engenharia Rural e Solos, UNESP. E-mails: maltoni@agr.feis.unesp.br; anamaria@bio.feis.unesp.br
} 


\title{
SUMMARY: CHEMICAL AND ORGANIC FERTILIZATION OF DEGRADED SOIL AND MYCORRHIZATION OF Stryphnodendron polyphyllum
}

\begin{abstract}
Due to the building of the Hydroelectric Power Plant of Ilha Solteira, in the late 60s, some areas have been deforested and were used as "loan areas". Thus, the soils belonging to these locations were subtracted and these areas' physical, chemical and biological properties became inappropriate. This work aimed at evaluating the use of chemical and organic fertilization to improve the degraded subsoil and replanting with mycorrhizal Stryphnodendron polyphyllum ("barbatimão"). The area is located at the Experimental Station (FEPE), UNESP/Ilha Solteira Campus, in Selvíria - MS. Using the design of randomized blocks with four replications of $250 \mathrm{~m}^{2}$ plots $(10 \times 25 \mathrm{~m}), 10$ treatments were evaluated: control; liming; fertilizing $N+P$; liming $+N+P ; N+P+$ water hyacinth; $N+P$ + sugar cane bagasse; $N+P+$ water hyacinth + sugar cane bagasse; liming $+N+P+$ water hyacinth; liming $+N+P+$ sugar cane bagasse; and liming $+N+P+$ water hyacinth + sugar cane bagasse. Chemical characteristics of the subsoil and plant growth were evaluated five times (June, August, November and December 2005, and March of 2006) and the root percent colonization was evaluated in March 2006. After a year the subsoil still remained acidic and of low chemical fertility. The steam diameter and plant height showed increasing results along the period, with the highest values for the treatments that received liming, $N+P$ and organic residues. The percent mycorrhizal colonization and number of spores were positively influenced by the presence of organic residues.
\end{abstract}

Index terms: loan area, savannah, water hyacinth, sugar cane bagasse.

\section{INTRODUÇÃO}

Área degradada é aquela que sofreu alteração de suas características originais, em decorrência de causas naturais ou oriundas de ação antrópica. Em algumas dessas áreas ocorre a eliminação dos meios bióticos, e estas apresentam baixa resiliência (Reichmann Neto, 1993; Crestana et al., 2006).

Com a construção de usinas hidrelétricas surgem áreas degradadas, como as "áreas de empréstimo", cuja caracterização química mostrou que o subsolo é pobre em nutrientes e matéria orgânica, além de apresentar propriedades físicas comprometidas e distantes das condições naturais, para o bioma Cerrado (Rodrigues et al., 2007).

Como medida mitigadora dos impactos ambientais, o uso da cobertura vegetal é uma opção prática, econômica e coerente na recuperação de áreas degradadas (Valcarcel \& D'Alterio, 1998). Dentre as espécies vegetais, as que possuem associações micorrízicas devem ser empregadas, visto que esses fungos desempenham importante papel na aquisição e mobilização de nutrientes do solo, promovendo ganhos no crescimento e desenvolvimento das plantas (Moreira \& Siqueira, 2006). Além do intuito de maximizar o processo de recuperação, houve a intenção de escolher plantas que promovam algum retorno econômico, como as espécies arbóreas do gênero Stryphnodendron, uma vez que possuem propriedades cicatrizantes de lesões gástricas, contra leishmaniose, e sua madeira pode ser usada na construção civil (Lorenzi, 2002). O barbatimão (Stryphnodendron polyphyllum Mart.), leguminosa pertencente à família
Fabaceae, tem sido recomendado para a composição de reflorestamentos mistos, visando à recuperação da vegetação de áreas degradadas e de preservação permanente (Souza \& Lorenzi, 2005).

Dessa forma, para restabelecer o potencial produtivo de um solo, equilibrar e sustentar o ecossistema, é necessário a elevação do teor da matéria orgânica. A sua redução afeta os processos de formação e estabilização dos agregados do solo, atividade biológica e ciclagem de nutrientes (Roscoe et al., 2006). Sua reintrodução ao solo ocorre por meio da adição de resíduos orgânicos. O aguapé (Eichhornia crassipes (Martius) Solms-Laubach)). é considerado a mais séria planta daninha aquática do País, com potencial de aumento de área de $15 \%$ ao dia; pode produzir $480 \mathrm{t} \mathrm{ha}^{-1}$ $\mathrm{ano}^{-1}$ de massa verde (Marcondes \& Tanaka, 1997). Sua biomassa é rica em macro e micronutrientes e normalmente utilizada como aditivo para solos erodidos, exauridos, desestruturados, desprovidos de matéria orgânica (Lutzenberger, 1985).

O Brasil hoje é considerado o principal produtor mundial de cana-de-açúcar (Saccharum sp.), a qual é considerada uma das plantas que possuem mecanismos fisiológicos mais aperfeiçoados, eficiência de assimilação de $\mathrm{CO}_{2}$ na fotossíntese e capacidade de produzir matéria verde (INCIDCA, 1999). O bagaço de cana é um subproduto da fabricação de açúcar e do álcool e vem sendo utilizado como matéria-prima na geração de energia elétrica (Paranhos, 1987).

Este trabalho objetivou avaliar os efeitos da aplicação da adubação química e orgânica na fertilidade do subsolo degradado e na micorrização do S. polyphyllum (barbatimão). 


\section{MATERIAL E MÉTODOS}

O experimento foi instalado em março/2005 e conduzido até abril/2006, em área degradada, localizada na Fazenda Experimental de Pesquisa e Extensão (FEPE) da UNESP-Universidade Estadual Paulista, Faculdade de Engenharia de Ilha Solteira, município de Selvíria - MS. O solo de maior ocorrência no local foi classificado como Latossolo Vermelho distrófico (Demattê, 1980).

Originalmente, a área apresentava como cobertura vegetal o Cerrado sensu stricto. No final da década de 1960, com a construção da Usina Hidrelétrica de Ilha Solteira, o local foi desmatado e uma parte dessa área foi utilizada como "área de empréstimo", de onde o solo foi retirado, variando de 8 a $12 \mathrm{~m}$ a profundidade de corte. Atualmente, nesse local, pequenas áreas estão em processo de regeneração natural, mas, em sua maior extensão, o subsolo permanece exposto, desprovido de cobertura vegetal e com presença de acentuados processos erosivos (Rodrigues et al., 2007).

Analisaram-se as características químicas e o número de esporos de fungos micorrízicos arbusculares (FMA) do subsolo da área degradada antes da instalação do experimento. Uma amostra, constituída de quatro amostras simples, foi coletada na profundidade de $0-0,15 \mathrm{~m}$, seca ao ar, peneirada $(2 \mathrm{~mm})$ e homogeneizada. Parte da amostra foi enviada para análise química para fins de fertilidade, segundo método proposto por Raij \& Quaggio (1983) (Quadro 1).

A outra parte da amostra de subsolo foi empregada para verificação do número de esporos de FMA autóctones. Para isso, $100 \mathrm{~g}$ da amostra foram processados segundo uma associação dos métodos de decantação e peneiramento úmido (Gerdemann \& Nicolson, 1963), seguidos de centrifugação e flutuação em sacarose (Jenkins, 1964), sendo encontrados 37 esporos.
O aguapé foi coletado manualmente e com auxílio de um gancho, em uma lagoa em área particular, próxima ao perímetro urbano de Ilha Solteira. O bagaço de cana-de-açúcar foi doado pela Usina Pioneiros, de Sud Mennucci - SP. A planta de aguapé e o bagaço de cana foram secos ao ar e triturados para redução e uniformização do tamanho (cerca de $1 \mathrm{~cm}$ de comprimento). Analisou-se uma amostra de cada material, determinando os teores de nutrientes, segundo Malavolta et al. (1997) (Quadro 2).

A espécie arbórea utilizada foi o $S$. polyphyllum. As mudas foram produzidas a partir de sementes coletadas na região, as quais foram escarificadas quimicamente com ácido sulfúrico concentrado. Estas foram germinadas em papel germi test e transplantadas para sacos plásticos de polipropileno, com capacidade para $2 \mathrm{~kg}$ de substrato. O subsolo da área degradada foi coletado na área do experimento, com correção da acidez, e adubado com N e P, conforme análise anteriormente realizada. Procedeu-se à rega diária em viveiro e, no qüinquagésimo dia, as mudas foram transferidas para o lado de fora deste, onde permaneceram por 10 dias, como forma de adaptá-las às condições de campo; no sexagésimo dia, quando atingiram $5 \mathrm{~cm}$ de altura em média, elas foram transplantadas para o campo.

Utilizou-se o delineamento em blocos ao acaso, com quatro parcelas de $250 \mathrm{~m}^{2}$ (10 x $\left.25 \mathrm{~m}\right)$. Essa área foi escarificada a $0,40 \mathrm{~m}$ de profundidade e gradeada. Foram abertas covas de $0,30 \mathrm{~m}$ de diâmetro por $0,90 \mathrm{~m}$ de profundidade, com broca hidráulica, no espaçamento de $2 \times 2 \mathrm{~m}$. O volume do subsolo da metade inferior da cova foi devolvido e os resíduos orgânicos foram misturados à metade do volume superior do subsolo de cada cova (cerca de $0,064 \mathrm{~m}^{3}$ ), na quantidade de $954 \mathrm{~g} /$ cova, equivalente a $30 \mathrm{t} \mathrm{ha}^{-1}$ de material seco (Schiaveto et al., 2003), conforme distribuição dos tratamentos, além da calagem (15,9 g/cova, equivalente a $497 \mathrm{~kg} / \mathrm{ha}$ de calcário, PRNT

Quadro 1. Características química do subsolo da área degradada antes da instalação do experimento

\begin{tabular}{|c|c|c|c|c|c|c|c|c|c|c|}
\hline $\mathbf{P}$ & MO & $\mathrm{pH} \mathrm{CaCl}_{2}$ & $\mathbf{K}$ & $\mathrm{Ca}^{2+}$ & $\mathrm{Mg}^{2+}$ & $\mathbf{H}+\mathbf{A l}$ & $\mathrm{Al}^{3+}$ & SB & СтC & $\mathrm{V}$ \\
\hline $\mathrm{mg} \mathrm{dm}^{-3}$ & $\mathrm{~g} \mathrm{dm}^{-3}$ & & & 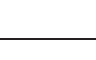 & 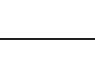 & $\mathrm{mmol}_{\mathrm{c}} \mathrm{dm}$ & & & & $\%$ \\
\hline 1 & 7 & 4,2 & 0,3 & 1 & 1 & 31 & 9 & 2,3 & 33,3 & 7 \\
\hline
\end{tabular}

Quadro 2. Composição química (teores totais) do aguapé e bagaço de cana-de-açúcar secos

\begin{tabular}{|c|c|c|c|c|c|c|c|c|c|c|c|}
\hline & $\mathbf{N}$ & $\mathbf{P}$ & $\mathbf{K}$ & $\mathbf{C a}$ & $\mathbf{M g}$ & $\mathbf{S}$ & B & $\mathrm{Cu}$ & $\mathrm{Fe}$ & Mn & $\mathrm{Zn}$ \\
\hline & \multicolumn{6}{|c|}{$-\mathrm{g} \mathrm{kg}^{-1}$} & \multicolumn{5}{|c|}{$-\mathrm{mg} \mathrm{kg}^{-1}$} \\
\hline Aguapé & 8,61 & 0,83 & 8,45 & 25,15 & 6,08 & 3,43 & 43,15 & 18,30 & 1.371 & 1.148 & 9,50 \\
\hline Bagaço de cana & 3,92 & 0,09 & 1,18 & 1,18 & 2,10 & 0,55 & 11,06 & 33,40 & 900 & 104 & 41,20 \\
\hline
\end{tabular}


82), adubação nitrogenada (12 g/cova equivalente a $375 \mathrm{~kg} \mathrm{ha}^{-1}$ de sulfato de amônio) e fosfatada (123,7 g/cova, equivalente a $3.865 \mathrm{~kg} \mathrm{ha}^{-1}$ de surperfosfato simples), como segue: 1) testemunha; 2) calagem; 3) adubação com $\mathrm{N}+\mathrm{P}$; 4) calagem $+\mathrm{N}+\mathrm{P}$; 5) $\mathrm{N}+\mathrm{P}+$ aguapé; 6) $\mathrm{N}+\mathrm{P}+$ bagaço de cana; 7$) \mathrm{N}+\mathrm{P}+$ aguapé + bagaço de cana; 8 ) calagem $+\mathrm{N}+\mathrm{P}+$ aguapé; 9) calagem + $\mathrm{N}+\mathrm{P}+$ bagaço de cana; e 10) calagem $+\mathrm{N}+\mathrm{P}+$ aguapé + bagaço de cana.

Durante o plantio das mudas (abril/2005) foram depositados na cova, a $0,10 \mathrm{~m}$ de profundidade, $50 \mathrm{~g}$ de solo proveniente de uma área de Cerrado preservado da FEPE, como fonte de microrganismos. Essa prática teve como objetivo introduzir e, ou, aumentar a população de espécies de microrganismos, importante em qualquer processo de recuperação, especialmente dos FMA.

Após o transplante das mudas em campo, elas foram irrigadas duas vezes por semana, utilizando um tanque-pipa, até o mês de outubro. Para aumentar a retenção de água, foi feito o coroamento nas covas, com raio de $0,60 \mathrm{~m}$, antes do plantio das mudas. A partir dos 60 dias após o plantio e com intervalos de 30 dias, promoveu-se capina no perímetro da coroa e entre linhas. Os dados pluviométricos do período de experimentação estão apresentados na figura 1 .

As amostragens do experimento para análise das características químicas e do crescimento das plantas foram realizadas em cinco épocas (junho, agosto, novembro e dezembro/2005 e março/2006). A coleta de raízes para avaliação da colonização micorrízica ocorreu apenas uma vez, durante a última coleta (março/2006). O crescimento das plantas foi verificado por meio da medição da altura, com uma régua de madeira, e do diâmetro do colo, com de um paquímetro. Foram avaliadas cinco plantas por repetição, por tratamento, em cada época de amostragem.

As amostras de subsolo foram compostas de cinco amostras simples, também oriundas de cinco plantas, determinadas aleatoriamente, por tratamento, por repetição, à profundidade de $0-0,10 \mathrm{~m}$, utilizando-se um trado tipo caneca. Essas amostras foram secas ao ar, peneiradas e homogeneizadas. Parte das amostras foi enviada para análise das características químicas do subsolo, como anteriormente descrito. Parte do subsolo da última amostragem foi reservada para contagem do número de esporos dos FMA, conforme método descrito previamente.

As raízes coletadas foram separadas do subsolo, lavadas em água corrente e preservadas temporariamente em álcool 50 \%. Para avaliação da colonização micorrízica (COL), as raízes foram novamente lavadas, clarificadas com KOH $10 \%$, acidificadas com $\mathrm{HCl} 1 \%$, coradas com azul de tripano 0,05\%, de acordo com (Phillips \& Hayman, 1970), e preservadas em lactoglicerol. Avaliaram-se 100 segmentos de raízes finas, com cerca de $1 \mathrm{~cm}$ de comprimento, por amostra, sob microscópio óptico (40x), em lâminas de vidro, sendo considerados infectados os que apresentavam arbúsculos e, ou, vesículas e, ou, hifas intracelulares.

$\mathrm{Na}$ análise dos dados considerou-se o modelo estatístico na forma de parcelas subdivididas, com os 10 tratamentos nas parcelas e as cinco épocas de amostragens como subparcelas, exceto para colonização micorrízica. Segundo o modelo adotado e para cada uma das variáveis, realizou-se a análise de variância (teste F), com as complementações por meio do teste de Scott-Knott nas comparações de médias de tratamentos e o estudo de regressão na análise de tendência ao longo das épocas de amostragens. Avaliaram-se também coeficientes de correlação linear. Foram utilizados os programas SISVAR (Ferreira, 1999-2003) e SAS (1999) nas análises.

\section{RESULTADOS E DISCUSSÃO}

Com relação a crescimento do barbatimão, diâmetro do colo (Quadro 3) e altura de planta (Quadro 4), observaram-se diferenças significativas entre os tratamentos, entre épocas de amostragens e na interação. Na terceira, quarta e quinta épocas de amostragem, os tratamentos 1 e 2 apresentaram os

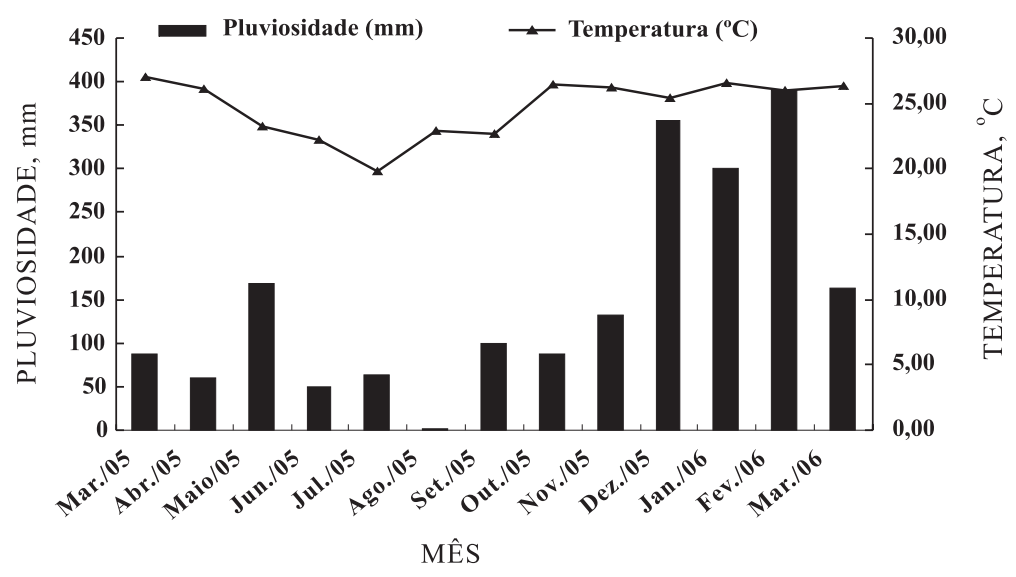

Figura 1. Médias de temperatura e precipitação para o período do experimento, compreendido entre março de 2005 e abril de 2006. Fontes: UNESP (2006). 
menores valores de diâmetro do caule na altura do colo e altura de planta. Na quinta época de amostragem, o tratamento 5 para diâmetro do caule na altura do colo e os tratamentos 5, 8 e 10 para altura de planta foram os que exibiram maiores valores - tratamentos esses com adição do resíduo orgânico aguapé.

Quadro 3. Diâmetro do caule na altura do colo do Stryphnodendron polyphyllum entre os tratamentos (Trat.) e as diferentes épocas de amostragem, com respectivos modelos de equação de regressão, significância para o valor de $\mathbf{F}$ para o coeficiente de maior grau $\mathbf{e ~}^{2}$

\begin{tabular}{|c|c|c|c|c|c|c|c|c|}
\hline \multirow{2}{*}{ Trat. } & \multicolumn{5}{|c|}{ Época de amostragem } & \multirow{2}{*}{ Modelo da equação } & \multirow{2}{*}{$\begin{array}{l}\text { Valor } \\
\text { de F }\end{array}$} & \multirow{2}{*}{$\mathrm{R}^{2}$} \\
\hline & Jun./05 & Ago./05 & Nov./05 & Dez./05 & Mar./06 & & & \\
\hline 1 & 4,71 & $5,17 \mathrm{~b}$ & $7,10 \mathrm{~b}$ & $8,40 \mathrm{c}$ & $8,98 \mathrm{e}$ & $\hat{\mathrm{Y}}=3,33775+1,17675 \mathrm{x}$ & $32,74^{* *}$ & 0,96 \\
\hline 2 & 4,98 & $5,61 \mathrm{~b}$ & $7,81 \mathrm{~b}$ & $8,90 \mathrm{c}$ & $10,45 \mathrm{e}$ & $\hat{\mathrm{Y}}=3,276+1,424 \mathrm{x}$ & $47,94^{* *}$ & 0,98 \\
\hline 3 & 7,35 & $8,70 \mathrm{a}$ & $13,94 \mathrm{a}$ & $16,61 \mathrm{a}$ & $20,98 \mathrm{~d}$ & $\hat{\mathrm{Y}}=3,9935+3,5005 \mathrm{x}$ & $289,72^{* *}$ & 0,98 \\
\hline 4 & 7,20 & $9,20 \mathrm{a}$ & $14,25 \mathrm{a}$ & $17,00 \mathrm{a}$ & $25,55 \mathrm{c}$ & $\hat{\mathrm{Y}}=1,2570+4,457 \mathrm{x}$ & $469,68^{* *}$ & 0,94 \\
\hline 5 & 6,53 & $8,30 \mathrm{a}$ & $15,50 \mathrm{a}$ & $18,24 \mathrm{a}$ & 30,85 a & $\hat{\mathrm{Y}}=6,9125-1,532821 \mathrm{x}+1,232679 \mathrm{x}^{2}$ & $50,29^{* *}$ & 0,97 \\
\hline 6 & 5,77 & $6,97 \mathrm{~b}$ & $12,29 \mathrm{a}$ & $14,64 \mathrm{~b}$ & $22,90 \mathrm{~d}$ & $\hat{\mathrm{Y}}=5,5045-0,584571 \mathrm{x}+0,796429 \mathrm{x}^{2}$ & $20,99^{* *}$ & 0,98 \\
\hline 7 & 5,66 & $7,83 \mathrm{a}$ & $14,00 \mathrm{a}$ & $17,65 \mathrm{a}$ & $28,20 \mathrm{~b}$ & $\hat{Y}=5,309-0,607571 x+1,016429 x^{2}$ & $34,19^{* *}$ & 0,99 \\
\hline 8 & 6,87 & $8,00 \mathrm{a}$ & $14,35 \mathrm{a}$ & $17,70 \mathrm{a}$ & $28,20 \mathrm{~b}$ & $\hat{\mathrm{Y}}=7,177-1,507714 \mathrm{x}+1,124286 \mathrm{x}^{2}$ & $41,84^{* *}$ & 0,98 \\
\hline 9 & 5,66 & $6,95 \mathrm{~b}$ & $12,50 \mathrm{a}$ & $14,65 \mathrm{~b}$ & $24,78 \mathrm{c}$ & $\hat{\mathrm{Y}}=6,254-1,519071 \mathrm{x}+1,018929 \mathrm{x}^{2}$ & $34,36^{* *}$ & 0,97 \\
\hline 10 & 5,84 & $6,90 \mathrm{~b}$ & $13,25 \mathrm{a}$ & $15,90 \mathrm{~b}$ & $26,30 \mathrm{~b}$ & $\hat{Y}=6,143-1,424357 x+1,069643 x^{2}$ & $37,87^{\star *}$ & 0,97 \\
\hline \multicolumn{3}{|c|}{ Valor de F para Trat x EA } & \multicolumn{2}{|c|}{$18,073 * *$} & & & & \\
\hline \multicolumn{3}{|l|}{ CV (\%) } & \multicolumn{2}{|c|}{10,23} & & & & \\
\hline
\end{tabular}

Médias seguidas de mesma letra, nas colunas, não diferem significativamente pela análise de Scott-Knott a $1 \%$. Tratamentos: 1) testemunha; 2) calagem; 3) adubação com $\mathrm{N}+\mathrm{P}$; 4) calagem $+\mathrm{N}+\mathrm{P}$; 5) $\mathrm{N}+\mathrm{P}+$ aguapé; 6) $\mathrm{N}+\mathrm{P}+$ bagaço de cana; 7) $\mathrm{N}+\mathrm{P}+$ aguapé + bagaço de cana; 8 ) calagem $+\mathrm{N}+\mathrm{P}+$ aguapé; 9) calagem $+\mathrm{N}+\mathrm{P}+$ bagaço de cana; e 10) calagem $+\mathrm{N}+\mathrm{P}+$ aguapé + bagaço de cana. ${ }^{* *}$, significativo a $1 \%$.

Quadro 4. Altura do Stryphnodendron polyphyllum, entre os tratamentos (Trat.) e as diferentes épocas de amostragem de subsolo, com respectivos modelos de equação de regressão, significância para o valor de $\mathbf{F}$ para o coeficiente de maior grau e $\mathbf{R}^{2}$

\begin{tabular}{|c|c|c|c|c|c|c|c|c|}
\hline \multirow{2}{*}{ Trat. } & \multicolumn{5}{|c|}{ Época de amostragem } & \multirow{2}{*}{ Modelo da equação } & \multirow{2}{*}{ Valor de F } & \multirow{2}{*}{$\mathbf{R}^{2}$} \\
\hline & Jun./05 & Ago./05 & Nov./05 & Dez./05 & Mar./06 & & & \\
\hline 1 & 8,63 & 10,60 & $13,88 \mathrm{~b}$ & $18,88 \mathrm{c}$ & $21,85 \mathrm{e}$ & $\hat{\mathrm{Y}}=4,3475+3,4725 \mathrm{x}$ & $14,39^{* *}$ & 0,98 \\
\hline 2 & 10,73 & 12,20 & $16,33 \mathrm{~b}$ & $22,50 \mathrm{c}$ & $28,65 \mathrm{e}$ & $\hat{Y}=4,235+4,615 x$ & $25,42^{* *}$ & 0,95 \\
\hline 3 & 18,53 & 24,38 & 39,75 a & $47,75 \mathrm{~b}$ & $52,28 \mathrm{~d}$ & $\hat{\mathrm{Y}}=9,2725+9,0875 \mathrm{x}$ & $98,58^{* *}$ & 0,96 \\
\hline 4 & 17,63 & 23,68 & $51,33 \mathrm{a}$ & $58,60 \mathrm{~b}$ & $79,90 \mathrm{~b}$ & $\hat{\mathrm{Y}}=-1,6175+15,9475 \mathrm{x}$ & $303,59^{* *}$ & $0 ., 96$ \\
\hline 5 & 16,70 & 25,33 & $46,53 \mathrm{a}$ & 69,46 a & 92,93 a & $\hat{Y}=6,9175+6,19625 x+2,24375 x^{2}$ & $8,41^{\text {** }}$ & 0,99 \\
\hline 6 & 12,63 & 18,63 & $43,85 \mathrm{a}$ & $53,98 \mathrm{~b}$ & $72,68 \mathrm{c}$ & $\hat{Y}=-6,285+15,545 x$ & $288,46^{* *}$ & 0,97 \\
\hline 7 & 14,10 & 19,18 & $48,50 \mathrm{a}$ & $67,00 \mathrm{a}$ & $87,00 \mathrm{~b}$ & $\hat{\mathrm{Y}}=-10,9325+19,3625 \mathrm{x}$ & $447,53^{* *}$ & 0,97 \\
\hline 8 & 16,00 & 20,25 & $48,35 \mathrm{a}$ & $63,90 \mathrm{a}$ & $105,20 \mathrm{a}$ & $\hat{\mathrm{Y}}=14,9-4,173571 \mathrm{x}+4,396429 \mathrm{x}^{2}$ & $32,30^{* *}$ & 0,98 \\
\hline 9 & 10,90 & 17,60 & $43,20 \mathrm{a}$ & $53,90 \mathrm{~b}$ & $84,73 \mathrm{~b}$ & $\hat{\mathrm{Y}}=3,555+4,102143 \mathrm{x}+2,382143 \mathrm{x}^{2}$ & $9,48^{* *}$ & 0,98 \\
\hline 10 & 12,85 & 17,30 & $44,95 \mathrm{a}$ & $58,10 \mathrm{~b}$ & 97,80 a & $\hat{Y}=10,99-2,93 x+4, x^{2}$ & $26,73^{* *}$ & 0,98 \\
\hline \multicolumn{3}{|c|}{ Valor de F para Trat x EA } & \multicolumn{2}{|c|}{$15,648^{* *}$} & & & & \\
\hline \multicolumn{3}{|c|}{$\mathrm{CV}(\%)$} & \multicolumn{2}{|c|}{14,76} & & & & \\
\hline
\end{tabular}

Médias seguidas de mesma letra, nas colunas, não diferem significativamente pela análise de Scott-Knott a $1 \%$. Tratamentos: 1) testemunha; 2) calagem; 3) adubação com $\mathrm{N}+\mathrm{P}$; 4) calagem $+\mathrm{N}+\mathrm{P}$; 5) $\mathrm{N}+\mathrm{P}+$ aguapé; 6$) \mathrm{N}+\mathrm{P}+$ bagaço de cana; 7) $\mathrm{N}+\mathrm{P}+$ aguapé + bagaço de cana; 8 ) calagem $+\mathrm{N}+\mathrm{P}+$ aguapé; 9) calagem $+\mathrm{N}+\mathrm{P}+$ bagaço de cana; e 10 ) calagem $+\mathrm{N}+\mathrm{P}+$ aguapé + bagaço de cana. ** significativo a $1 \%$. 
O diâmetro do caule e a altura de planta apresentaram, de maneira geral, resultados crescentes ao longo do período, sendo os maiores valores verificados nos tratamentos que receberam calagem associada a $\mathrm{N}+\mathrm{P}$ e resíduos orgânicos. A partir da terceira época de amostragem essas diferenças passaram a ser significativas para altura de planta, coincidindo com o período de maior pluviosidade. Os tratamentos que receberam calagem, $\mathrm{N}+\mathrm{P}$ e resíduos orgânicos, associados ou não, foram os que proporcionaram os maiores valores destas duas variáveis (Quadros 3 e 4).

Em um experimento de produção de mudas de Acacia mearnsii, obtiveram-se os maiores valores de altura de planta e diâmetro de caule quando foi utilizada a casca de Pinus sp. decomposta misturada a resíduo vegetal no substrato, associada a doses de vermicomposto e vermiculita (Caldeira et al., 2000). Observaram-se, também, os maiores incrementos em altura para a espécie arbórea Astronium fraxinifolium nos tratamentos que continham o resíduo orgânico misturado ao substrato (Silva et al., 2005). No presente trabalho, o tratamento 3 , seguido do 1 e do 6 , mostrou valores elevados de saturação por $\mathrm{Al}^{3+}$. Com isso, pode-se ratificar a relação entre os teores de
$\mathrm{Al}^{3+}$ e o crescimento de planta, ou seja, os maiores crescimentos ocorreram onde os valores de saturação por $\mathrm{Al}^{3+}$ foram menores (Quadro 5). Correlação negativa e significativa foi observada entre $\mathrm{Al}^{3+}$ e altura de planta (Quadro 6)

O elevado índice de acidez e excesso de $\mathrm{Al}^{3+}$, levando à baixa aquisição de nutrientes e água, torna as culturas sujeitas às deficiências minerais e suscetíveis a déficits hídricos e, conseqüentemente, proporciona baixo crescimento de planta (Maria et al., 1993). Neste trabalho, observou-se, entre diâmetro do caule e altura de planta, correlação significativa e positiva (Quadro 6).

Da mesma forma, avaliando diversos tipos de substrato e suas associações para produção de mudas da espécie Cybistax antisyphilitica, Pereira et al. (2005) encontraram maior crescimento quando utilizaram solo de Cerrado e resíduo orgânico na proporção 3:1, respectivamente, resultando em plantas com maior altura, diâmetro do caule e matéria fresca de raiz.

Após um ano da aplicação dos tratamentos, observaram-se diferenças estatísticas quanto às características químicas do subsolo entre os

Quadro 5. Médias, valores de F e coeficientes de variação para as características químicas do subsolo, coletado na profundidade de $\mathbf{0}-\mathbf{0 , 1 0} \mathrm{m}$, para os diferentes tratamentos épocas de amostragens de subsolo (EA)

\begin{tabular}{|c|c|c|c|c|c|c|c|c|c|c|c|}
\hline Trat./EA & $\mathbf{P}$ & MO & pH $\mathrm{CaCl}_{2}$ & $\mathbf{K}$ & $\mathrm{Ca}^{2+}$ & $\mathrm{Mg}^{2+}$ & $\mathbf{H}+\mathbf{A l}$ & $\mathrm{Al}^{3+}$ & SB & CTC & $\mathbf{V}$ \\
\hline & $\mathrm{mg} \mathrm{dm}^{-3}$ & $\mathrm{~g} \mathrm{dm}^{-3}$ & & & & 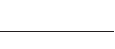 & $\mathrm{nmol}_{\mathrm{c}} \mathrm{dm}$ & & & & $\%$ \\
\hline 1 & $1,60 \mathrm{c}$ & $2,15 \mathrm{~b}$ & $4,90 \mathrm{~d}$ & $0,60 \mathrm{c}$ & $3,00 \mathrm{c}$ & $2,15 \mathrm{c}$ & $19,30 \mathrm{a}$ & $2,00 \mathrm{~b}$ & $5,65 \mathrm{c}$ & $24,95 \mathrm{c}$ & $22,60 \mathrm{c}$ \\
\hline 2 & $1,60 \mathrm{c}$ & $2,10 \mathrm{~b}$ & $5,00 \mathrm{c}$ & $0,35 \mathrm{c}$ & $7,25 \mathrm{~b}$ & $3,30 \mathrm{~b}$ & $17,05 \mathrm{~b}$ & $0,75 \mathrm{c}$ & $10,85 \mathrm{~b}$ & $27,90 \mathrm{~b}$ & $38,10 \mathrm{~b}$ \\
\hline 3 & $2,10 \mathrm{c}$ & $2,10 \mathrm{~b}$ & $4,90 \mathrm{~d}$ & $0,05 \mathrm{c}$ & $3,50 \mathrm{c}$ & $2,10 \mathrm{c}$ & $20,50 \mathrm{a}$ & $2,95 \mathrm{a}$ & $5,80 \mathrm{c}$ & $26,30 \mathrm{c}$ & $22,10 \mathrm{c}$ \\
\hline 4 & $2,40 \mathrm{~b}$ & $2,80 \mathrm{~b}$ & $5,00 \mathrm{c}$ & $0,30 \mathrm{c}$ & $6,15 \mathrm{~b}$ & $2,55 \mathrm{c}$ & $17,55 \mathrm{~b}$ & $1,10 \mathrm{c}$ & $9,35 \mathrm{~b}$ & $26,90 \mathrm{c}$ & $34,60 \mathrm{~b}$ \\
\hline 5 & $2,00 \mathrm{c}$ & $4,70 \mathrm{a}$ & $5,05 \mathrm{~b}$ & $1,75 \mathrm{a}$ & $6,05 \mathrm{~b}$ & $3,65 \mathrm{~b}$ & $17,00 \mathrm{~b}$ & $0,40 \mathrm{~d}$ & $11,60 \mathrm{~b}$ & $28,60 \mathrm{~b}$ & $40,00 \mathrm{~b}$ \\
\hline 6 & $2,00 \mathrm{c}$ & $4,65 \mathrm{a}$ & $4,95 \mathrm{~d}$ & $1,45 \mathrm{~b}$ & $3,85 \mathrm{c}$ & $2,50 \mathrm{c}$ & $19,25 \mathrm{a}$ & $2,25 \mathrm{~b}$ & $7,00 \mathrm{c}$ & $26,25 \mathrm{c}$ & $26,05 \mathrm{c}$ \\
\hline 7 & $2,20 \mathrm{c}$ & $5,85 \mathrm{a}$ & $5,00 \mathrm{c}$ & $1,75 \mathrm{a}$ & $5,85 \mathrm{~b}$ & $3,65 \mathrm{~b}$ & $16,95 \mathrm{~b}$ & $0,80 \mathrm{c}$ & $11,10 \mathrm{~b}$ & $28,60 \mathrm{~b}$ & $38,20 \mathrm{~b}$ \\
\hline 8 & $2,95 \mathrm{a}$ & $5,10 \mathrm{a}$ & $5,45 \mathrm{a}$ & $2,00 \mathrm{a}$ & $9,70 \mathrm{a}$ & $4,90 \mathrm{a}$ & $15,30 \mathrm{c}$ & $0,15 \mathrm{~d}$ & $16,60 \mathrm{a}$ & $31,90 \mathrm{a}$ & $50,50 \mathrm{a}$ \\
\hline 9 & $2,10 \mathrm{c}$ & $5,15 \mathrm{a}$ & $5,00 \mathrm{c}$ & $1,20 \mathrm{~b}$ & $6,60 \mathrm{~b}$ & $3,30 \mathrm{~b}$ & $17,95 \mathrm{~b}$ & $1,00 \mathrm{c}$ & $10,55 \mathrm{~b}$ & $28,50 \mathrm{~b}$ & $36,35 \mathrm{~b}$ \\
\hline 10 & $2,40 \mathrm{~b}$ & $5,20 \mathrm{a}$ & $5,05 \mathrm{~b}$ & $1,40 \mathrm{~b}$ & $6,85 \mathrm{~b}$ & $3,75 \mathrm{~b}$ & $16,80 \mathrm{~b}$ & $0,45 \mathrm{~d}$ & $11,95 \mathrm{~b}$ & $29,25 \mathrm{~b}$ & $40,70 \mathrm{~b}$ \\
\hline Valor de F & $4,762^{* * *}$ & $18,876^{* \star}$ & $14,751^{\text {** }}$ & $9,24^{* *}$ & $10,981^{* *}$ & $10,628^{* \star}$ & $10,556^{* *}$ & $18,213^{* *}$ & $12,989^{* *}$ & $5,458^{* *}$ & $20,108^{* *}$ \\
\hline CV (\%) & 38,13 & 38,92 & 6,32 & 83,59 & 46,16 & 37,55 & 11,80 & 80,84 & 40,72 & 13,27 & 25,54 \\
\hline Jun./05 & 1,35 & 4,08 & 5,00 & 1,15 & 7,23 & 3,83 & 18,28 & 1,83 & 12,28 & 30,80 & 37,83 \\
\hline Ago./05 & 1,33 & 4,38 & 5,05 & 1,28 & 6,20 & 3,18 & 16,35 & 1,25 & 10,28 & 26,63 & 37,48 \\
\hline Nov./05 & 2,00 & 3,70 & 5,13 & 1,13 & 5,30 & 2,93 & 18,10 & 0,53 & 9,28 & 27,65 & 33,15 \\
\hline Dez./05 & 3,00 & 4,15 & 5,98 & 1,08 & 5,35 & 3,13 & 18,20 & 0,83 & 9,45 & 27,65 & 33,23 \\
\hline Mar./06 & 3,00 & 3,60 & 5,00 & 0,80 & 5,33 & 2,88 & 17,90 & 1,53 & 8,45 & 26,85 & 32,93 \\
\hline Valor de F & $39,579^{* *}$ & $4,178^{\star *}$ & $17,987^{\text {*k }}$ & $2,805^{\star}$ & $7,135^{\text {** }}$ & $10,035^{\star *}$ & $22,55^{* \star}$ & $10,7825^{* *}$ & $10,305^{\star \star}$ & $16,427^{\star *}$ & $8,844^{* *}$ \\
\hline C V (\%) & 39,30 & 25,09 & 3,07 & 69,07 & 33,91 & 23,81 & 6,02 & 44,25 & 26,09 & 9,14 & 15,21 \\
\hline $\begin{array}{l}\text { Modelo } \\
\text { equação }\end{array}$ & $\mathrm{C}$ & $\mathrm{L}$ & $\mathrm{Q}$ & $\mathrm{L}$ & $\mathrm{Q}$ & $\mathrm{C}$ & $\mathrm{C}$ & $\mathrm{Q}$ & $\mathrm{Q}$ & $\mathrm{C}$ & $\mathrm{L}$ \\
\hline $\mathrm{R}^{2}(\%)$ & 99,54 & 33,13 & 94,58 & 75,53 & 98,14 & 97,77 & 76,08 & 93,03 & 96,52 & 94,85 & 79,11 \\
\hline Valor de F & $1,232 \mathrm{~ns}$ & $1,505 \mathrm{~ns}$ & $1,477 \mathrm{~ns}$ & $1,081^{\mathrm{ns}}$ & $0,709 \mathrm{~ns}$ & $1,233 \mathrm{~ns}$ & $1,119 \mathrm{~ns}$ & $1,805^{* *}$ & $0,883^{\mathrm{ns}}$ & $0,832^{\mathrm{ns}}$ & $0,875^{\mathrm{ns}}$ \\
\hline
\end{tabular}

Médias seguidas de mesma letra, nas colunas, não diferem significativamente pela análise de Scott-Knott a $1 \%$. Tratamentos: 1) testemunha; 2) calagem; 3) adubação com $\mathrm{N}+\mathrm{P}$; 4) calagem $+\mathrm{N}+\mathrm{P}$; 5) $\mathrm{N}+\mathrm{P}+$ aguapé; 6) $\mathrm{N}+\mathrm{P}+$ bagaço de cana; 7) $\mathrm{N}+\mathrm{P}+$ aguapé + bagaço de cana; 8 ) calagem $+\mathrm{N}+\mathrm{P}+$ aguapé; 9 ) calagem $+\mathrm{N}+\mathrm{P}+$ bagaço de cana; e 10 ) calagem $+\mathrm{N}+\mathrm{P}+$ aguapé + bagaço de cana. **, * significativo a 1 e 5 \%; ns: não-significativo. L: linear; Q: quadrática; e C: cúbica. 
Quadro 6. Coeficiente de correlação para diâmetro do caule (Diam.), altura da planta (Alt.), características químicas da análise de solo, número de esporos (Esp.) e colonização micorrízica (Col.)

\begin{tabular}{|c|c|c|c|c|c|c|c|c|c|c|c|c|c|c|}
\hline & Diam. & Alt. & $\mathbf{P}$ & MO & $\begin{array}{c}\text { pH } \\
\mathrm{CaCl}\end{array}$ & $\mathbf{K}$ & $\mathbf{C a}^{2+}$ & $\mathbf{M g}^{2+}$ & $\mathbf{H}+\mathbf{A l}$ & $\mathrm{Al}^{3+}$ & SB & CTC & V & Esp. \\
\hline Alt. & $0,96^{* *}$ & & & & & & & & & & & & & \\
\hline $\mathrm{P}$ & $0,62^{* *}$ & $0,62^{* *}$ & & & & & & & & & & & & \\
\hline MO & 0,08 & $0,13^{*}$ & $0,18^{* *}$ & & & & & & & & & & & \\
\hline $\mathrm{pH}$ & 0,12 & $0,17^{*}$ & $0,26^{* *}$ & $0,46^{* *}$ & & & & & & & & & & \\
\hline K & $-0,04$ & $-0,00$ & 0,07 & $0,59^{* *}$ & $0,41^{* *}$ & & & & & & & & & \\
\hline $\mathrm{Ca}^{2+}$ & $-0,06$ & $-0,03$ & $0,15^{*}$ & $0,38^{* *}$ & $0,62^{* *}$ & $0,29^{* *}$ & & & & & & & & \\
\hline $\mathrm{Mg}^{2+}$ & $-0,08$ & $-0,03$ & $0,14^{*}$ & $0,51^{* *}$ & $0,63^{* *}$ & $0,47^{* *}$ & $0,73^{* *}$ & & & & & & & \\
\hline $\mathrm{H}+\mathrm{Al}$ & $-0,02$ & $-0,03$ & $-0,10$ & $-0,39^{* *}$ & $-0,71^{\star *}$ & $-0,35^{\star \star}$ & $-0,49^{* *}$ & $-0,50^{* *}$ & & & & & & \\
\hline $\mathrm{Al}^{3+}$ & $-0,12$ & $-0,19^{* *}$ & $-0,24^{* *}$ & $-0,37^{* *}$ & $-0,75^{* *}$ & $-0,35^{\text {** }}$ & $-0,43^{* *}$ & $-0,45^{* *}$ & $0,66^{* *}$ & & & & & \\
\hline SB & $-0,06$ & $-0,02$ & $0,17^{* *}$ & $0,52^{* *}$ & $0,70^{* *}$ & $0,43^{* *}$ & $0,95^{* *}$ & $0,87^{* *}$ & $-0,55^{* *}$ & $-0,50^{* *}$ & & & & \\
\hline CTC & $-0,10$ & $-0,05$ & 0,12 & $0,39^{* *}$ & $0,42^{* *}$ & $0,32^{* *}$ & $0,83^{* *}$ & $0,76^{* *}$ & $-0,11$ & $-0,20^{* *}$ & $0,86^{* *}$ & & & \\
\hline $\mathrm{V}$ & $-0,00$ & 0,03 & $0,15^{*}$ & $0,53^{* *}$ & $0,78^{* *}$ & $0,44^{* *}$ & $0,90^{* *}$ & $0,82^{* *}$ & $-0,71^{* *}$ & $-0,64^{* *}$ & $0,95^{* *}$ & $0,71^{* *}$ & & \\
\hline Esp. & $0,46^{\star \star \star}$ & $0,48^{* * k}$ & 0,00 & 0,19 & 0,18 & 0,19 & 0,08 & 0,20 & $-0,22$ & $-0,19$ & 0,16 & 0,15 & 0,19 & \\
\hline Col. & 0,13 & 0,16 & 0,27 & $0,77^{* *}$ & $0,43^{* *}$ & $0,46^{* *}$ & $0,36^{* *}$ & $0,48^{* *}$ & $-0,51^{* *}$ & $-0,43^{* *}$ & $0,48^{* *}$ & $0,44^{* *}$ & $0,44^{*}$ & $0,38^{*}$ \\
\hline
\end{tabular}

*,**: significativo a 1 e $5 \%$, respectivamente.

tratamentos e épocas de amostragem, com relação às variáveis analisadas (Quadro 5). O subsolo continuou a apresentar caráter ácido e pobreza em nutrientes, podendo ser considerado típico de região de Cerrado e de área degradada (Malavolta \& Kliemann, 1985), mesmo após ter sido submetido aos tratamentos. Entretanto, verificou-se sensível aumento nos valores de $\mathrm{P}, \mathrm{K}, \mathrm{Ca}^{2+}, \mathrm{Mg}^{2+}$, SB, CTC e V e diminuição nos valores de $\mathrm{MO}(\mathrm{H}+\mathrm{Al})$, e $\mathrm{Al}^{3+}$ quando comparados aos valores anteriores à instalação do experimento (Quadro 1).

O teor de $\mathrm{P}$ disponível apresentou o maior valor para o tratamento $8 \mathrm{e}$, durante as épocas de amostragem, exibiu aumentos crescentes dos valores. Os valores de $\mathrm{P}$ refletem sua disponibilização e concentração no substrato (Silva et al., 2002). No presente trabalho, benefícios foram proporcionados pelos resíduos vegetais, especialmente o aguapé, o qual apresentou valores de $P$ quase 10 vezes superiores aos do bagaço-de-cana (Quadro 2), além da presença de microrganismos, responsáveis pela ciclagem de nutrientes, que regulam as transformações do resíduo orgânico e a disponibilização de nutrientes, como o P (Turco et al., 1994).

A variável MO mostrou diferenças entre os tratamentos que receberam e os que não receberam resíduos orgânicos (Quadro 5), sendo possível inferir a contribuição dos resíduos utilizados para elevar o teor desta no subsolo. A MO pode proporcionar a estabilização dos agregados do solo, aumentando sua resistência à erosão e sua capacidade de retenção de água (Silva et al., 2002). O aguapé, um material que se decompõe rapidamente e contém alto teor de água, é um composto rico em macro e micronutrientes, o que o torna um resíduo orgânico de boa qualidade (Lutzenberger, 1985).
Ao serem associados dois ou mais resíduos orgânicos, deve-se procurar melhor equilíbrio na relação C/N. Assim, utiliza-se, normalmente, material rico em $\mathrm{N}$ associado ao material rico em $\mathrm{C}$ orgânico (Silva et al., 2002). Neste trabalho, o aguapé mostrou melhorar as condições químicas do solo, como pode ser verificado no tratamento com adição de $\mathrm{N}+\mathrm{P}$ mais bagaço e aguapé (tratamento 7), quando comparado ao sem aguapé (tratamento 6), possivelmente devido à sua qualidade nutricional (Quadro 5) e por promover equilíbrio na relação $\mathrm{C} / \mathrm{N}$ entre os resíduos, facilitando a decomposição.

Ao longo do experimento, no entanto, detectou-se comportamento decrescente dos teores de MO. Observações semelhantes a essas foram relatadas em estudo de Silva et al. (2005), onde, com a adição de resíduos orgânicos (lodo de esgoto), eram esperados incrementos nos teores de $\mathrm{MO}$, o que não aconteceu de modo satisfatório. Os autores atribuem o ocorrido ao fato de os resíduos aplicados apresentarem efeitos efêmeros, ou seja, a MO ter sido mineralizada ao longo do experimento. No presente trabalho, no entanto, para MO, não foram verificadas diferenças estatísticas significativas entre épocas, em 12 meses de experimentação.

Quanto ao pH, constataram-se diferenças significativas entre os tratamentos (Quadro 5), sendo os maiores valores proporcionados pelos tratamentos 8, 5 e 10, ou seja, os que possuíam em comum adubação com $\mathrm{N}$ e $\mathrm{P}$ e aguapé. Ao longo do experimento, os valores de $\mathrm{pH}$ se comportaram de maneira quadrática, com diminuição no final. Essa curta duração do efeito da calagem pode ser explicada pelo caráter ácido do subsolo. $\mathrm{O}$ aumento nos valores de $\mathrm{pH}$, segundo Silva et al. (2002), pode estar relacionado à liberação de grupamentos aniônicos provenientes da quebra de 
proteínas e polissacarídeos, gerando grupamentos $\mathrm{R}$ $\mathrm{COOH}, \mathrm{R}-\mathrm{OH}$ e Ø-OH (fenólicos), representando assim a tendência de humificação do material orgânico em decomposição e à calagem.

O K apresentou os maiores teores nos tratamentos 5, 7 e 8, os quais possuíam em comum a adição de resíduos orgânicos (aguapé e, ou, bagaço de cana), adubação com $\mathrm{N}$ e $\mathrm{P}$ e calagem para o tratamento 8, e a variável apresentou ajuste linear (Quadro 5). $\mathrm{O} \mathrm{Ca}^{2+}$ e o $\mathrm{Mg}^{2+}$ exibiram comportamento semelhante entre si, com os maiores valores no tratamento 8 , que associou a calagem, a adubação química e o aguapé (Quadro 5). Incrementos em $\mathrm{Ca}^{2+}$ também foram relatados por Silva et al. (2005) em estudo dos efeitos da rochagem e da adubação orgânica sobre o crescimento de uma espécie arbórea do cerrado. Esses autores constataram os maiores valores nos tratamentos que receberam aguapé seco e triturado, adicionado ao solo.

Quanto à acidez potencial $(\mathrm{H}+\mathrm{Al})$, os maiores valores foram observados nos tratamentos 1,3 e 6 , os quais tinham em comum a ausência da calagem. Pode-se afirmar que a calagem contribuiu para a redução da $(\mathrm{H}+\mathrm{Al})$, associado ou não à adição dos resíduos orgânicos, com exceção do tratamento 6 (Quadro 5).

Com relação a SB, CTC e V, verificou-se comportamento semelhante entre os tratamentos e ao longo das épocas de amostragem do subsolo (Quadro 5). Os maiores valores foram verificados no tratamento 8 , que recebeu calagem, adubação e aguapé, enquanto os tratamentos 1, 3 e 6, que não receberam calagem, apresentaram valores menores. Observou-se ajuste linear para V e quadrático para SB e CTC. A recomendação do $\mathrm{V}$ para culturas de reflorestamento, segundo Raij et al. (1997), deve ser de $50 \%$. O presente trabalho apresentou os tratamentos com valores inferiores a este, exceto o tratamento 8; além de valores baixos para $\mathrm{Mg}^{2+}$, médio para $\mathrm{K}, \mathrm{pH}$ e $\mathrm{V}$ e alto para $\mathrm{Ca}^{2+}$. Esses resultados podem ser explicados pelos efeitos da calagem como promotora da diminuição da acidez, insolubilização e complexação de elementos tóxi-

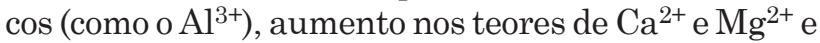
disponibilização de $\mathrm{P}$, além dos efeitos favoráveis à microflora do subsolo que atua nos resíduos vegetais, no caso, o aguapé.

Quanto ao $\mathrm{Al}^{3+}$ (Quadro 5), os tratamentos 2, 4, 7, 8, 9 e 10 mostraram menores valores de saturação por $\mathrm{Al}^{3+}$, possivelmente justificado pela associação da calagem (exceto para o 7) com os resíduos orgânicos. Houve diferenças significativas para interação entre tratamentos e épocas de amostragem de subsolo (Quadro 7), com os valores menores verificados nos tratamentos 5; 8 e 10, os quais receberam calagem e resíduos orgânicos. Esse comportamento condiz com o relatado por Miyazawa et al. (2000), os quais mostram que a presença de resíduos orgânicos reduz a acidez do perfil do solo, com decréscimo na saturação por $\mathrm{Al}^{3+}$ e aumento da eficiência no aproveitamento dos nutrientes pelas plantas.

Quadro 7. Alumínio trocável em diferentes épocas de amostragens de subsolo (EA), coletado na profundidade de 0-0,10 m e respectivos modelos de equação de regressão, significância para o valor de $F$ para $o$ coeficiente de maior grau e $\mathbf{R}^{2}$

\begin{tabular}{|c|c|c|c|c|c|c|c|c|}
\hline \multirow[b]{2}{*}{ Trat. } & \multicolumn{5}{|c|}{ Época de amostragem de subsolo } & \multirow[b]{2}{*}{ Equação } & \multirow{2}{*}{$\begin{array}{l}\text { Valor } \\
\text { de F }\end{array}$} & \multirow[b]{2}{*}{$\mathbf{R}^{2}$} \\
\hline & Jun./05 & Ago./05 & Nov./05 & Dez./05 & Mar./06 & & & \\
\hline & & $-\mathrm{Al}(\mathrm{n}$ & $\mathrm{mol}_{\mathrm{c}} \mathrm{dm}^{-3}$ & 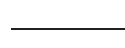 & - & & & \\
\hline 1 & $2,75 \mathrm{~b}$ & $2,25 \mathrm{~b}$ & $1,25 \mathrm{a}$ & $2,00 \mathrm{a}$ & $1,75 \mathrm{~b}$ & $\hat{\mathrm{Y}}=3,8-1,189286 \mathrm{x}+0,160714 \mathrm{x}^{2}$ & $5,26^{*}$ & 0,69 \\
\hline 2 & $1,50 \mathrm{c}$ & $0,50 \mathrm{c}$ & $0,25 \mathrm{~b}$ & $0,75 \mathrm{~b}$ & $0,75 \mathrm{c}$ & $\hat{\mathrm{Y}}=2,5-1,303571 \mathrm{x}+0,196429 \mathrm{x}^{2}$ & $7,85^{* *}$ & 0,79 \\
\hline 3 & $3,75 \mathrm{a}$ & $3,75 \mathrm{a}$ & $1,50 \mathrm{a}$ & $2,25 \mathrm{a}$ & $3,50 \mathrm{a}$ & $\hat{\mathrm{Y}}=2,45+2,851190 \mathrm{x}-1,669643 \mathrm{x}^{2}+0,229167 \mathrm{x}^{3}$ & $11,00^{* *}$ & 0,79 \\
\hline 4 & $2,25 \mathrm{~b}$ & $1,00 \mathrm{c}$ & $0,50 \mathrm{~b}$ & $0,50 \mathrm{~b}$ & $1,25 \mathrm{~b}$ & $\hat{\mathrm{Y}}=4,1-2,178571 \mathrm{x}+0,321429 \mathrm{x}^{2}$ & $21,03^{* *}$ & 0,99 \\
\hline 5 & $0,75 \mathrm{c}$ & $0,00 \mathrm{~d}$ & $0,00 \mathrm{~b}$ & $0,00 \mathrm{~b}$ & $1,25 \mathrm{~b}$ & $\hat{\mathrm{Y}}=2,1-1,614286 \mathrm{x}+0,285714 \mathrm{x}^{2}$ & $16,62^{* *}$ & 0,93 \\
\hline 6 & $3,25 \mathrm{a}$ & $2,25 \mathrm{~b}$ & $1,25 \mathrm{a}$ & $1,75 \mathrm{a}$ & $2,75 \mathrm{a}$ & $\hat{\mathrm{Y}}=5,45-2,507143 \mathrm{x}+0,392857 \mathrm{x}^{2}$ & $31,42^{* *}$ & 0,95 \\
\hline 7 & $1,25 \mathrm{c}$ & $1,00 \mathrm{c}$ & $0,00 \mathrm{~b}$ & $0,50 \mathrm{~b}$ & $1,25 \mathrm{~b}$ & $\hat{\mathrm{Y}}=2,7-1,55 \mathrm{x}+0,25 \mathrm{x}^{2}$ & $12,72^{* *}$ & 0,76 \\
\hline 8 & $0,50 \mathrm{c}$ & $0,00 \mathrm{~d}$ & $0,00 \mathrm{~b}$ & $0,00 \mathrm{~b}$ & $0,25 \mathrm{c}$ & $\mathrm{ns}$ & - & - \\
\hline 9 & $1,50 \mathrm{c}$ & $1,00 \mathrm{c}$ & $0,50 \mathrm{~b}$ & $0,50 \mathrm{~b}$ & $1,50 \mathrm{~b}$ & $\hat{Y}=2,9-1,55 x+0,25 x^{2}$ & $12,72^{* *}$ & 0,90 \\
\hline 10 & $0,75 \mathrm{c}$ & $0,75 \mathrm{c}$ & $0,00 \mathrm{~b}$ & $0,00 \mathrm{~b}$ & $0,75 \mathrm{c}$ & $\hat{\mathrm{Y}}=1,8-1,039286 \mathrm{x}+0,160714 \mathrm{x}^{2}$ & $5,26^{*}$ & 0,61 \\
\hline \multicolumn{4}{|c|}{ Valor de F para Tratx EA } & \multicolumn{2}{|c|}{$1,805^{* *}$} & & & \\
\hline \multicolumn{3}{|c|}{$\mathrm{CV}(\%)$} & & \multicolumn{2}{|c|}{44,25} & & & \\
\hline
\end{tabular}

Médias seguidas de mesma letra, nas colunas, não diferem significativamente pela análise de Scott-Knott a $1 \%$. Tratamentos: 1) testemunha; 2) calagem; 3) adubação com $\mathrm{N}+\mathrm{P}$; 4) calagem $+\mathrm{N}+\mathrm{P}$; 5) $\mathrm{N}+\mathrm{P}+$ aguapé; 6$) \mathrm{N}+\mathrm{P}+$ bagaço de cana; 7) $\mathrm{N}+\mathrm{P}+$ aguapé + bagaço de cana; 8 ) calagem $+\mathrm{N}+\mathrm{P}+$ aguapé; 9 ) calagem $+\mathrm{N}+\mathrm{P}+$ bagaço de cana; e 10$)$ calagem $+\mathrm{N}+\mathrm{P}+$ aguapé + bagaço de cana. **, * significativo a 1 e $5 \%$, respectivamente. 
A colonização micorrízica e o número de esporos de FMA mostraram diferenças significativas entre tratamentos (Quadro 8). A colonização micorrízica foi verificada em todos os tratamentos, com os maiores valores encontrados nos tratamentos $5,6,7,8,9$ e 10, os quais apresentam em comum a adubação associada à adição de resíduos orgânicos, que, provavelmente, propiciaram condição favorável à inoculação com solo de uma área de cerrado preservado, realizada no plantio das mudas de barbatimão.

No cultivo em áreas de revegetação, Pereira \& Oliveira (2005) encontraram as maiores percentagens de colonização micorrízica em Stryphnodendron adstringens que cresceu na área com maior quantidade de resíduo orgânico, quando comparado a sistemas convencionais de cultivo. Resultados semelhantes foram observados quando se avaliou a percentagem de colonização em mudas de Coffea arabica (Siqueira et al., 1995), Peltogyne venosa e Sclerolobium paniculatum (Caldeira et al., 1999), com as maiores alturas das plantas nos tratamentos cujo substrato foi inoculado com esporos de fungos micorrízicos.

No presente trabalho, a colonização micorrízica variou de 70 a $84 \%$, sendo considerada alta. Valores semelhantes foram também encontrados para

Quadro 8. Médias, valores de F e coeficientes de variação $(\mathrm{CV})$ determinados para colonização micorrízica e número de esporos de fungos micorrízicos arbusculares (FMA)

\begin{tabular}{ccc}
\hline Tratamento & $\begin{array}{c}\text { Colonização } \\
\text { micorrizica }\end{array}$ & $\begin{array}{c}\text { No de esporos } \\
\text { de FMA }\end{array}$ \\
\hline & $\%$ & $100 \mathrm{~g}^{-1}$ solo seco \\
1 & $71,25 \mathrm{~b}$ & $48,75 \mathrm{c}$ \\
2 & $71,50 \mathrm{~b}$ & $52,75 \mathrm{c}$ \\
3 & $70,75 \mathrm{~b}$ & $168,50 \mathrm{~b}$ \\
4 & $70,00 \mathrm{~b}$ & $210,25 \mathrm{~b}$ \\
5 & $83,00 \mathrm{a}$ & $335,00 \mathrm{a}$ \\
6 & $83,75 \mathrm{a}$ & $166,50 \mathrm{~b}$ \\
7 & $84,25 \mathrm{a}$ & $193,00 \mathrm{~b}$ \\
8 & $81,75 \mathrm{a}$ & $148,00 \mathrm{~b}$ \\
9 & $79,00 \mathrm{a}$ & $90,75 \mathrm{c}$ \\
10 & $81,50 \mathrm{a}$ & $143,25 \mathrm{~b}$ \\
Valor de $\mathrm{F}$ & 7,441 ** & $10,796{ }^{* *}$ \\
CV (\%) & 5,17 & 32,78 \\
\hline
\end{tabular}

Médias seguidas de mesma letra, nas colunas, não diferem significativamente pela análise de Scott-Knott a $1 \%$. Tratamentos: testemunha; calagem; adubação com $\mathrm{N}+\mathrm{P}$; calagem $+\mathrm{N}+\mathrm{P} ; \mathrm{N}+\mathrm{P}+$ aguapé; $\mathrm{N}+\mathrm{P}+$ bagaço de cana; $\mathrm{N}+\mathrm{P}+$ aguapé + bagaço de cana; calagem $+\mathrm{N}+\mathrm{P}+$ aguapé; calagem $+\mathrm{N}+\mathrm{P}+$ bagaço de cana; e calagem $+\mathrm{N}+\mathrm{P}+$ aguapé + bagaço de cana. ${ }^{* *}$ significativo a $1 \%$.
S. polyphyllum, $86 \%$ (Pereira \& Oliveira, 2005), e em diversas espécies arbóreas que crescem em solos do território cearense, com colonização média de 77 \% (Vasconcelos et al., 1984). Benefícios da associação micorrízica foram relatados por Zangaro et al. (2002), que, estudando espécies arbóreas nativas da bacia do rio Tibati no Paraná, detectaram a importância da inoculação com fungos micorrízicos das mudas destinadas a solos degradados e pobres em nutrientes.

Acredita-se que os valores intermediários de $\mathrm{pH}$ e os baixos teores de $\mathrm{P}$ não interferiram negativamente na atividade dos fungos, mas estimularam, de certa forma, a micorrização, tendo em vista relatos de que elevados teores de $\mathrm{P}$ reduzem a infecção (Daft \& Nicolson, 1969).

Quanto ao número de esporos, o tratamento 5 exibiu o maior valor (335), o qual foi cerca de nove vezes superior à quantidade de esporos que havia inicialmente na área. Assim como no presente trabalho, Caproni et al. (2003), estudando a ocorrência de FMA em áreas revegetadas após mineração, constataram que, tanto a quantidade quanto a variedade de espécies de esporos foram significativamente menores em áreas não-revegetadas ou com subsolo estéril.

\section{CONCLUSÕES}

1. O subsolo continua a apresentar caráter ácido e pobreza de nutrientes, após um ano de avaliação.

2. O crescimento da planta foi sistemático ao longo do período, com os maiores valores para os tratamentos que receberam calagem, adubação e resíduos orgânicos, especialmente o aguapé.

3. A colonização micorrízica foi influenciada positivamente pela presença de resíduos orgânicos e o número de esporos, pela presença de aguapé.

\section{LITERATURA CITADA}

CALDEIRA, M.V.W.; SCHUMACHER, M.V. \& TEDESCO, N. Crescimento de mudas de Acacia mearnsii em função de diferentes doses de vermicomposto. Sci. Flor., 57:161-170, 2000 .

CALDEIRA, M.V.W.; SILVA, E.M.R.; FRANCO, A.A. \& ZANAN, M.L.B. Efeito de fungos micorrízicos arbusculares no desenvolvimento de duas leguminosas arbóreas. Ci. Flor., 9:63-70, 1999

CAPRONI, A.L.; FRANCO, A.A.; BERBARA, R.L.L.; TRUFEM, S.B.; GRANHA, J.R.D.O. \& MONTEIRO, A.B. Ocorrência de fungos micorrízicos arbusculares em áreas revegetadas após mineração de bauxita em Porto Trombetas, Pará Pesq. Agropec. Bras., 38:1409-1418, 2003. 
CRESTANA, M.S.M.; FERRETTI, A.R.; TOLETO FILHO, D.V.; ARBOCZ, G.F.; SHIMIDT, H.A. \& GUARDIA, J.F.C. Espécies arbóreas nativas do Estado de São Paulo recomendadas para reflorestamentos. In: CRESTANA, M.S.M.; FERRETTI, A.R.; TOLETO FILHO, D.V.; ARBOCZ, G.F.; SHIMIDT, H.A. \& GUARDIA, J.F.C. Florestas: Sistemas de recuperação com essências nativas, produção de mudas e legislação. São Paulo, Imprensa oficial, 2006. p.49-84.

DAFT, M.J. \& NICOLSON, T.H. Effect of Endogone mycorrhiza on plant growh. II - Influence of soluble phosphate on endophyte and host in maize. New Phytol., 68:945-952, 1969 .

DEMATTÊ, J.L.I. Levantamento detalhado dos solos do "Campus experimental de Ilha Solteira". Piracicaba, Escola Superior Agrícola Luiz de Queiroz, 1980. 119p.

FERREIRA, D.F. SISVAR. Lavras, Universidade Federal de Lavras, 1999-2003. (Versão 4.2)

GERDEMANN, J.W. \& NICOLSON, T.H. Spores of mycorrhizal Endogone species extracted from soil by wet sieving and decanting. Trans. Br. Mycol. Soc., 46:234244, 1963.

INSTITUTO CUBANO DE PESQUISA DOS DERIVADOS DA CANA-DE-AÇÚCAR - INCIDCA. Manual dos derivados da cana-de-açúcar: diversificação, matérias primas, derivados do bagaço, derivados do melaço, outros derivados, resíduos, energia. Brasília, ABIPTI, 1999. 474p.

JENKINS, W.R. A rapid centrifugal-flotation technique for separating nematodes from soil. Plant Dis. Rep., 48:692, 1964.

LORENZI, H. Árvores brasileiras. 4.ed. Nova Odessa, Instituto Plantarum, 2002. v.2. p.204.

LUTZENBERGER, J. Em defesa do aguapé. 1985. Disponível em: <http://www.fgaia.org.br/texts/t-aguape.html> Acesso em: 23 set. de 2004.

MALAVOLTA, E. \& KLIEMANN, H.J. Desordens nutricionais no Cerrado. Piracicaba, Associação Brasileira para Pesquisa da Potassa e do Fosfato, 1985. 136p.

MALAVOLTA, E.; VITTI, G.C. \& OLIVEIRA, S. Avaliação do estado nutricional das plantas: Princípios e aplicações. 2.ed. Piracicaba, Potafos, 1997. 319p.

MARCONDES, D.A.S. \& TANAKA, R.H. Plantas aquáticas nos reservatórios das usinas hidrelétricas das CESP. In CONGRESSO BRASILEIRO DA CIÊNCIA DAS PLANTAS DANINHAS: WORKSHOP DE PLANTAS DANINHAS AQUÁTICAS, 21., Caxambu, 1997. Anais. Caxambu, Sociedade Brasileira da Ciência das Plantas Daninhas, 1997. p.2-4.

MARIA, I.C.; ROSSETTO, R.; AMBROSANO, E.J.; CASTRO, O.M. \& NEPTUNE, A.M.L. Efeito da adição de diferentes fontes de cálcio no movimento de cátions em colunas de solo. Sci. Agric., 50:87-98, 1993.

MIYAZAWA, M.; PAVAN, M.A. \& FRANCHINI, J.C. Neutralização da acidez do perfil do solo por resíduos vegetais. Piracicaba, Associação Brasileira para Pesquisa da Potassa e do Fosfato, 2000. 16p. (Informações Agronômicas, 92)
MOREIRA, F.M.S. \& SIQUEIRA, J.O. Microbiologia e bioquímica do solo. 2.ed. Lavras, Universidade Federal de Lavras, 2006. 729p.

PARANHOS, S.B. Cana de açúcar: Cultivo e utilização. Campinas, Fundação Cargill, 1987.v.2. 856p.

PEREIRA, J.A.A. \& OLIVEIRA, C.A. Efeitos do Eucalyptus camaldulensis sobre a colonização micorrízica e a nodulação em Dimorphandra mollis e Stryphnodendron adstringens, em Brasilândia, Minas Gerais. Cerne, 11:409415,2005 .

PEREIRA, L.B.; MALTONI, K.L.; CASTILHO, R.M.M. \& CASSIOLATO, A.M.R. Influência de compostos orgânicos no desenvolvimento de mudas de Cybistax antisyphilitica (Ipê caroba de flor verde). In: SIMPÓSIO NACIONAL, 6; CONGRESSO LATINO AMERICANO DE RECUPERAÇÃO DE ÁREAS DEGRADADAS, 1., Curitiba, 2005. A fauna em foco. Curitiba, SOBRADE, 2005. CD-ROM.

PHILLIPS, J.M. \& HAYMAN, D.S. Improved procedures for clearing roots and staining parasitic and vesiculararbuscular mycorrhizal fungi for rapid assessment of infection. Trans. Br. Mycol. Soc., 55:158-161, 1970.

RAIJ, B.van. \& QUAGGIO, J.A. Métodos de análises de solos para fins de fertilidade. Campinas, Instituto Agronômico de Campinas, 1983. 31p.

RAIJ, B.van.; CANTARELLA, H.; QUAGGIO, J.A. \& FURLANI, A.M.C. Recomendações de adubação e calagem para o Estado de São Paulo. Campinas, Instituto Agronômico de Campinas, 1997. 285p.

REICHMANN NETO, F. Recuperação de áreas degradadas na Região Sul. In: CONGRESSO FLORESTAL PANAMERICANO, 1., CONGRESSO FLORESTAL BRASILEIRO, 7., Curitiba, 1993. Anais. Curitiba, SBS/ SBEF, 1993. p.102-107.

RODRIGUES, G.B.; MALTONI, K.L. \& CASSIOLATO, A.M.R. Dinâmica da regeneração do subsolo de áreas degradadas dentro do bioma Cerrado. Agriambi, 11:73-80, 2007.

ROSCOE, R.; BOADEY, R.M. \& SALTON, J.C. Sistema de manejo e matéria orgânica do solo. In: ROSCOE, R.; MERCANTE, F.M. \& SALTON, J.C. Dinâmica da matéria orgânica do solo em sistemas conservacionistas. Dourados, Embrapa, 2006. p.17-42.

SAS Institute. SAS/STAT Procedure guide for personal computers. 9.ed. Cary, 1999. 334p.

SCHIAVETO, A.R.; MALTONI, K.L. \& CASSIOLATO, A.M.R. Utilização do aguapé como fonte de matéria orgânica e nutrientes na recuperação de áreas degradadas. In: CONGRESSO DE INICIAÇÃO CIENTÍFICA DA UNESP, 15., Marília, 2003. Anais. Marília, Universidade Estadual de São Paulo, 2003. CD ROM.

SILVA, C.D.; COSTA, L.M.; MATOS, A.T.; CECON, P.R. \& SILVA, D.D. Vermicompostagem de lodo de esgoto urbano e bagaço de cana-de-açúcar. Agriambi, 6:487-491, 2002. 
SILVA, E.A.; MATONI, K.L. \& CASSIOLATO, A.M.R. Efeito da rochagem e da adubação orgânica sobre o desenvolvimento de uma espécie arbórea de cerrado. In: CONGRESSO LATINO AMERICANO SOBRE RECUPERAÇÃO DE ÁREAS DEGRADADAS; SIMPÓSIO NACIONAL SOBRE RECUPERAÇ̃̃O DE ÁREAS DEGRADADAS, 6., Curitiba, 2005. Anais. Curitiba, SOBRADE/UFPR, 2005. CD-ROM.

SIQUEIRA, J.O.; SAGGIN-JUNIOR, O.J.; COLOZZI-FILHO, A. \& OLIVEIRA, E. Influência do substrato de formação e da micorriza no crescimento de mudas de cafeeiro transplantadas. Pesq. Agropec. Bras., 30:1417-1425, 1995.

SOUZA, V.C. \& LORENZI, H. Botânica sistemática: Guia ilustrado para identificação das famílias de Angiospermas da flora brasileira, baseado em APG II. Nova Odessa, Instituto Plantarum, 2005. 640p.

TURCO, R.F.; KENNEDY, A.C. \& JAWSON, M.D. Microbial indicators of soil quality. In: DORAN. J.W.; COLEMAN, D.C.; BEZDICEK, D.F. \& STEWART, B.A., eds. Defining soil quality for a sustainable environment. Madison, Soil Science Society of America, 1994. p.73-90.
UNIVERSIDADE ESTADUAL PAULISTA - UNESP. Departamento de Fitossanidade, Engenharia Rural e Solos/Área de Hidráulica e Irrigação. Dados climáticos de Ilha Solteira. Disponível em: <http//www.agr.feis.unesp.br/ clima.php>. Acesso em: 23 out. de 2006.

VALCARCEL, R. \& D'ALTERIO, C.F.V. Medidas fisicobiológicas de recuperação de áreas degradadas: Avaliação das modificações edáficas e fitossociológicas. R. Flor. Amb., 5:68-88, 1998.

VASCONCELOS, I.; ALMEIDA, R.T. \& MENDES FILHO, P.F. Ocorrência de rizóbios e endomicorrizas em leguminosas arbustivas do Estado do Ceará, Brasil. Ci. Agr., 1-2:45-52, 1984.

ZANGARO, W.; NISIZAKI, S.M.A.; DOMINGOS, J.C.B. \& NAKANO, E.M. Micorriza arbuscular em espécies arbóreas nativas da bacia do Rio Tibagi, Paraná. Cerne, 1:77-87, 2002.b 